\title{
Importance of sonography of the skin and subcutaneous tissue in the early diagnosis of melanoma in-transit metastasis with the presentation of two cases
}

\author{
Anna Czarnecka ${ }^{1,2,3}$, Roman Czarnecki ${ }^{1}$, Wojciech Witkiewicz ${ }^{1,2}$, Anita Hryncewicz-Gwóźdż ${ }^{4}$ \\ ${ }^{1}$ Regional Specialist Hospital, Research and Development Centre, Wroclaw, Poland \\ 2Foundation of Medical Innovations WROVASC, Wroclaw, Poland \\ ${ }^{3}$ Department of Cosmetology, Faculty of Physiotherapy, University School of Physical Education, Wroclaw, Poland \\ ${ }^{4}$ Department of Dermatology, Venerology and Allergology, Wroclaw Medical University, Wroclaw, Poland
}

Adv Dermatol Allergol 2018; XXXV (2): 204-207

DOI: https://doi.org/10.5114/ada.2018.75244

Sonography is a non-invasive and inexpensive diagnostic imaging method, widely used in the field of internal medicine, especially oncology. Penetration into the tissue and organs varies depending on the frequency of the ultrasound applied. For studying internal organs and lymph nodes, a frequency range of $7.5-15 \mathrm{MHz}$ is used, while for examining skin thickness, high-frequency scanners (HFUS) emitting waves at 20-100 MHz are applied [1]. The HFUS were first used in 1979. Since the 1980s, this method has constantly been improved and is now used for examining the thickness of skin lesions in different diseases - inflammatory diseases with fibrosis, psoriasis and lichen planus - and it is used to monitor the effectiveness of treating these conditions. High-frequency scaners are also used in cosmetic dermatology to assess skin damage [1, 2]. However, the most important use of sonography is in the diagnosis of cancer. While sonography cannot give a precise diagnosis of whether a skin lesion is malignant or not, nor specify the type of neoplasm, the test is nonetheless useful for assessing the depth of tumour infiltration. There was concordance between the results of ultrasound and histological tests in assessing the melanoma infiltration depth with the use of $20 \mathrm{MHz}$ [3-6]. In the case of very thin metastatic melanomas, where the infiltration depth is less than $1 \mathrm{~mm}$, it is necessary to apply a frequency of $100 \mathrm{MHz}$. The continued development of techniques using ultrasound, and in particular the introduction of colour Doppler sonography, which exposes the angiogenesis in a tumour, has also enabled the metastatic potential to be evaluated [7-9].

Sonography is also the most sensitive technique for evaluating the regional lymph nodes for the primary staging of routine surveillance and for the detection of melanoma recurrences. Moreover, it has recently been pointed out that 7.5 to $15 \mathrm{MHz}$ sonography is helpful in detecting metastases into the soft tissue in proximity of the primary melanoma tumour. According to the literature, this method is superior to physical examination in the detection of melanoma in-transit metastasis - metastases to the skin and subcutaneous tissue in the area of regional lymphatic drainage [10-17].

The paper presents two female patients with a malignant melanoma (MM) who, during sonography, were diagnosed with in-transit metastases to the subcutaneous tissue, which had been overlooked in physical examinations. Early diagnosis of the locoregional dissemination made it possible to use palliative surgery consisting of removing the metastatic lesions.

Case 1. In 2008, a woman aged 58 years had undergone surgery because of an MM located on her lower right leg. The cancer had a diameter of $2 \mathrm{~cm}$; histological test results showed the following: Clark III; Breslow $4 \mathrm{~mm}$ and BRAF mutation negative. Immunohistochemical test results were as follows: Vimentin (+), MelanA $(+)$ and $\mathrm{HMB}(+)$. There were no neoplastic changes in the sentinel lymph node biopsy or distant metastasis to the internal organs. Following the resection of the primary tumour, the patient was examined every 3 months over a period of 2 years and then every 6 months, during which the postoperative scar and the regional lymph nodes were evaluated by means of palpation and $\mathrm{MHz} 8$ ultrasound. Once a year a chest $\mathrm{X}$-ray and abdominal ultrasound were performed. Three years after the removal of the primary tumour, a recurrence in the postoperative scar occurred with a diameter of $10 \mathrm{~mm}$, which was removed surgically. Next, due to the disease's recurrence, an increased frequency of monitoring using

Address for correspondence: Anna Czarnecka, Regional Specialist Hospital, Research and Development Centre, 48 Okrężna St, 53-008 Wroclaw, Poland, fax: +48 501242 924, e-mail: anna.czarnecka30@onet.pl Received: 18.02.2017, accepted: 9.05.2017. 
$8 \mathrm{MHz}$ sonography was established in order to evaluate the regional recurrences in the skin and subcutaneous tissue. Just 3 months after the removal of melanoma recurrences, a sonography found seven hypoechogenic foci, 3-4 $\mathrm{mm}$ in diameter, located in the subcutaneous tissue of the lower right leg along the saphenous vein. All were removed surgically, and their neoplastic origin was confirmed by histological tests. A treatment using Dacarbazine was initiated and then stopped after 6 months due to bad tolerance of this medication by the patient. During the eight consecutive follow-up checks over a 2-year period, the sonography of the lower right extremity revealed single metastases in-transit (Figure 1), which were removed surgically. Five years after the removal of the primary tumour there occurred a massive dissemination of the neoplastic process in the form of 16 in-transit metastases to the subcutaneous tissue. The tumours were visible only through ultrasound; they were not clinically palpable. Due to the large number of metastatic lesions instead of surgical resections, electrochemotherapy was applied to the lower right extremity using cisplatin. After another 6 months, a physical examination of the lower right leg found two extensive metastases to the skin. Due to the difficult operating conditions hindering surgery, a tumour cryotherapy was performed. Over the next 2 years, sonography of the skin and subcutaneous tissue detected in-transit metastases ranging from one to thirteen lesions, with a diameter of 3-9 $\mathrm{mm}$, which did not show up in the physical examination. All were surgically removed. During the entire period of observation, no distant metastases were detected by the diagnostic images - X-ray of the chest, ultrasound of the abdomen and positron emission tomography (PET). The patient has been under observation since 2008, and is in generally good condition.

Case 2. In March 2014, a 69-year-old woman was operated on because of melanoma with three satellite metastases to the skin, located in the lower left leg. The primary tumour had a diameter of $5 \mathrm{~cm}$. Histopathological tests showed the following: Clark V, Breslow $15 \mathrm{~mm}$, PT-4a, angio-invasion features and perineural infiltration. Immunohistochemical tests were as follows: Vimentin (+) MelanA (+) HMB (+). BRAF mutations were negative. There were no neoplastic changes in the sentinel lymph node biopsy or distant metastases to the internal organs. The neoplasm was excised with a wide margin and a full thickness graft put in place of the removed skin. Similar to patient 1, after surgery, follow-up check-ups were carried out every 3 months, during which the postoperative scar and the regional lymph nodes were examined by palpation and an $8 \mathrm{MHz}$ ultrasound. Further, an $8 \mathrm{MHz}$ ultrasound was performed on the skin and subcutaneous tissue in the area of the neoplastic lesions. Once a year a chest X-ray and an abdominal ultrasound were performed. Nine months after the primary tumour surgery, in proximity of the postoperative scar, an $8 \mathrm{MHz}$ ultrasound revealed three nodules with diameters of 4, 5 and $8 \mathrm{~mm}$ located in the subcutaneous tissue (Figure 2); these were not detectable by means of physical examination. The nodules were removed surgically and histopathological tests confirmed that they were melanoma metastases. The patient was not referred for systemic treatment due to her advanced age. After 9 more months, the ultrasound diagnosed six nodules with diameters of 3-9 $\mathrm{mm}$, located subcutaneously within the lower left leg. In addition, a PET examination showed an increased uptake of FDG [1] due to high levels of metabolism in the skin of the lower left leg and within the pancreas and duodenum. Histopathological tests confirmed that they were melanoma metastases in duodenum. A surgical treatment of metastases to the abdominal cavity was considered; however, due to the overall bad health of the patient, who had had a heart attack and a coronary artery stenting 2 weeks prior to the detection of the metastases, the operation was abandoned. However, palliative surgical treatment of the metastases to the subcutaneous tissue was continued. Six melanoma metastases located in the subcutaneous tissue of the lower left leg were removed surgically.

Malignant melanoma is a skin tumour originating from melanocytes; its successful treatment depends on early diagnosis and surgical resection. The tumour exhibits a poor response to chemotherapy or radiotherapy. In cases where there is a dissemination of the neoplastic process, a palliative therapy is initiated, involving the excision of metastases and the use of systemic treatment. Dacarbazine, anti-CTLA4 monoclonal antibodies inhibiting the systemic mechanisms of immunosuppression in order to induce anti-tumour responses (T-cell activation), and molecularly targeted therapy with the inhibitors of serine-threonine kinases are used. Treatment strategies have been developed for early detection of satellite metastases to the skin, in-transit metastases (metastases located in the skin and subcutaneous tissue within the local lymphatic drainage) as well as metastases to lymph nodes and internal organs. The guidelines as to the frequency of controls in subsequent years after a surgery and the types of tests conducted during follow-ups differ slightly between countries. Patients are recommended to do self-checks, and medical check-ups are performed, from 1 to 6 per year, including the following: sonography of the lymph nodes and abdomen, chest X-ray, computed tomography $(\mathrm{CT})$, magnetic resonance imaging (MRI), positron emission tomography (PET), blood tests including complete blood count, creatinine levels, a liver function test, lactate dehydrogenase levels and $\beta$-S100 $[18,19]$. In oncology centers in Poland, check-ups are made every 3 to 4 months during the first 2 years after a primary melanoma surgery, then every 6 months for the next 3 years and then once a year. Particular attention is given to the assessment of regional lymphatic drainage and occurrences of in-transit metastases. In 


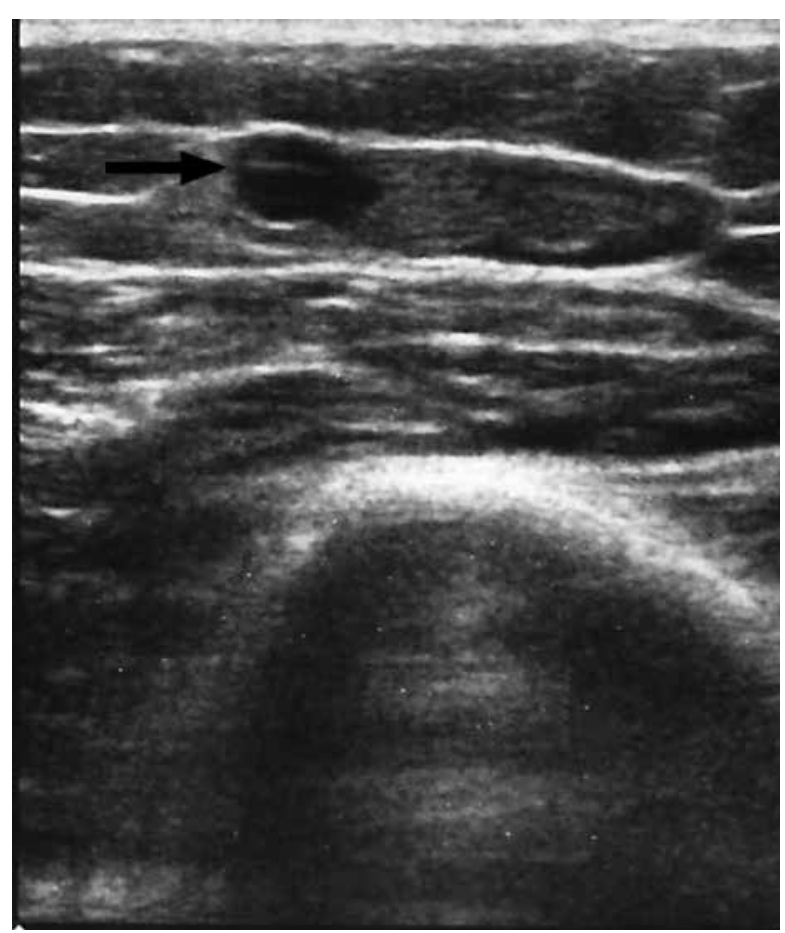

Figure 1. Ultrasound (8 MHz) imaging of in-transit metastasis of melanoma into the subcutaneous tissue (patient 1)

many countries, the sonography of regional lymph nodes is recommended in the MM follow-up guidelines as it improves survival rates [20]. However, there are no guidelines for sonography of the skin and subcutaneous tissue. Isolated reports in medical literature emphasize the usefulness of such an examination during the initial diagnosis as well as in the follow-up tests in the detection of in-transit metastases [21, 22]. Even small metastases to subcutaneous tissue with diameters of less than $10 \mathrm{~mm}$, which give no physical symptoms, can be observed as hypoechogenic foci using sonography [23-25]. Therefore, the test allows early detection and surgical removal of new, neoplastic metastatic lesions. In addition, the use of sonography, which allows for the accurate determination of the size and depth of a tumour, increases the precision of a surgical procedure and thus reduces the extent of necessary resections, which affects the quality of life of patients. After an early detection of metastases to the subcutaneous tissue, a minor surgery under local anaesthesia is carried out on an outpatient basis in order to remove the metastases. In addition, the use of sonography and early detection of melanoma dissemination could accelerate the implementation of systemic treatment. In both cases presented in the article, an $8 \mathrm{MHz}$ sonography of the skin and subcutaneous tissue was carried out by a surgical oncologist during the first visit and during each follow-up visit. The ultrasound examination of our patients has allowed for early detection of metastatic MM

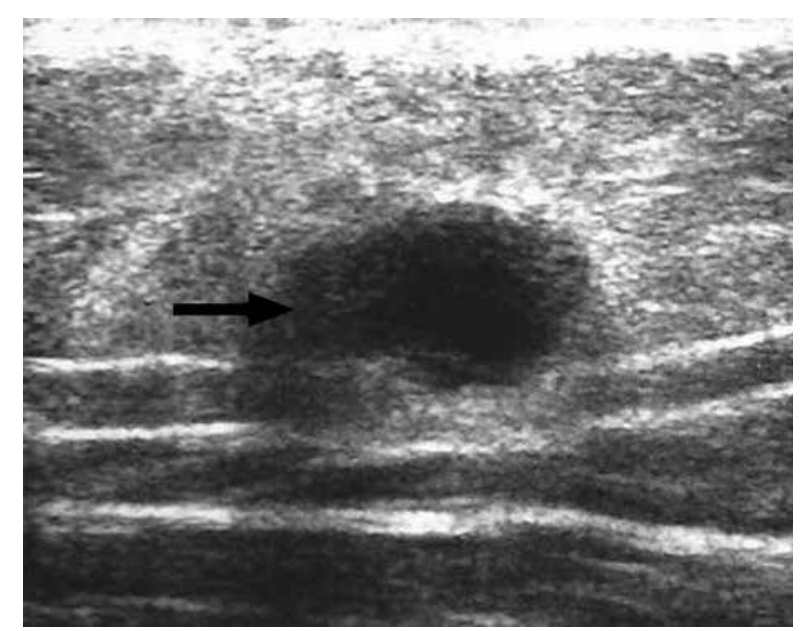

Figure 2. Ultrasound $(8 \mathrm{MHz})$ imaging of in-transit metastasis of melanoma into the subcutaneous tissue (patient 2)

to the subcutaneous tissue which had not been exposed in the clinical examination. As a result, the metastases were removed surgically or electrochemotherapy was performed early, when the lesions were small in diameter. Ultrasound examination showed nodules as little as $3 \mathrm{~mm}$ in diameter. Cutting out lesions as small as those was simple because of surgical techniques. It was also of little discomfort to the patient, while the healing time after the surgery was short. Early detection of the metastatic dissemination accelerated the implementation of systemic therapy in patient 1.

At present, ultrasound of the skin and subcutaneous tissue of patients with MM is not a standard examination. Due to a small number of reported cases where sonography was used for diagnosis of MM metastasis in-transit, it seems that this method has not yet found widespread use in follow-up procedures. We believe that this is a promising technique, non-invasive and inexpensive, allowing tumour staging to be accurately performed during the initial diagnosis. Furthermore, applied in a complementary way with standard screening tests, it allows for early diagnosis of local recurrences. Apart from radiologists, the training of specialists such as surgeons, oncologists and dermatologists who cooperate with oncology centers - in sonography of the skin and subcutaneous tissue will increase access to a valuable diagnostic method for patients with melanoma. We believe that the importance of sonography of the skin and subcutaneous tissue in patients with melanoma will continue to increase as clinicians gain experience in its use.

\section{Conflict of interest}

The authors declare no conflict of interest. 


\section{References}

1. Jasaitiene D, Valiukeviciene S, Linkeviciute G, et al. Principles of high-frequency ultrasonography for investigation of skin pathology. J Eur Acad Dermatol Venereol 2011; 25: 375-82.

2. Gambichler T, Moussa G, Bahrenberg K, et al. Preoperative ultrasonic assessment of thin melanocytic skin lesions using a 100-MHz ultrasound transducer: a comparative study. Dermatol Surg 2007; 33: 818-24.

3. Serrone L, Solivetti FM, Thorel MF, et al. High frequency ultrasound in the preoperative staging of primary melanoma: a statistical analysis. Melanoma Res 2002; 12: 287-90.

4. Kaikaris V, Valiukeviciene S, Rimdeika R. Sentinel lymph node biopsy in melanoma patients: methods, indications, and clinical significance. Medicina (Kaunas) 2003; 39: 621-30.

5. Pellacani G, Seidenari S. Preoperative melanoma thickness determination by 20-MHz sonography and digital videomicroscopy in combination. Arch Dermatol 2003; 139: 293-8.

6. Machet L, Belot V, Naouri M, et al. Preoperative measurement of thickness of cutaneous melanoma using highresolution $20 \mathrm{MHz}$ ultrasound imaging: a monocenter prospective study and systematic review of the literature. Ultrasound Med Biol 2009; 35: 1411-20.

7. Lassau N, Lamuraglia M, Koscielny S, et al. Prognostic value of angiogenesis evaluated with high-frequency and colour Doppler sonography for preoperative assessment of primary cutaneous melanomas: correlation with recurrence after a 5 year follow-up period. Cancer Imaging 2006; 25: 24-9.

8. Lassau N, Mercier S, Koscielny S, et al. Prognostic value of high-frequency sonography and color Doppler sonography for the preoperative assessment of melanomas. AJR Am J Roentgenol 1999; 172: 457-61.

9. Srivastava A, Laidler P, Davies RP, et al. The prognostic significance of tumor vascularity in intermediate-thickness (0.76-4.0 mm thick) skin melanoma. A quantitative histologic study. Am J Pathol 1988; 13: 419-23.

10. Prayer L, Winkelbauer H, Gritzmann N, et al. Sonography versus palpation in the detection of regional lymph-node metastases inpatients with malignant melanoma. Eur J Cancer 1990; 26: 827-30.

11. Binder M, Kittler H, Steiner A, et al. Lymph node sonography versus palpation for detecting recurrent disease in patients with malignant melanoma. Eur J Cancer 1997; 33: 1805-8.

12. Blum A, Schlagenhauff B, Stroebel W, et al. Ultrasound examination of regional lymph nodes significantly improves early detection of locoregional metastases during follow-up of patients with cutaneous melanoma: results of a prospective study of 1288 patients. Cancer 2000; 88: 2534-9.

13. Saiag P, Bernard M, Beauchet A, et al. Ultrasonography using simple diagnostic criteria vs palpation for the detection of regional lymph node metastases of melanoma. Arch Dermatol 2005; 141: 183-9.

14. Rossi CR, Seno A, Vecchiato A, et al. The impact of ultrasound scanning in the staging and follow-up of patients with clinical stage I cutaneous melanoma. Eur J Cancer 1997; 33: 200-3.

15. Brountzos EN, Panagiotou IE, Bafaloukos DI, et al. Ultrasonographic detection of regional lymph node metastases in patients with intermediate or thick malignant melanoma. Oncol Rep 2003; 10: 505-10.

16. Voit C, Proebstle TM, Winter H, et al. Presurgical ultrasoundguided anchor-wire marking of soft tissue metastases in stage III melanoma patients. Dermatol Surg 2001; 27: 129-32.

17. Machet L, Nemeth-Normand F, Giraudeau B, et al. Is ultrasound lymph node examination superior to clinical exami- nation in melanoma follow-up? A monocentre cohort study of 373 patients. Br J Dermatol 2005; 152: 66-70.

18. Voss RK, Woods TN, Cromwell KD, et al. Improving outcomes in patients with melanoma: strategies to ensure an early diagnosis. Patient Relat Outcome Meas 2015; 6: 229-42.

19. Trotter SC, Sroa N, Winkelmann RR, et al. A global review of melanoma follow-up guidelines. J Clin Aesthet Dermatol 2013; 6: 18-26.

20. Xing Y, Bronstein Y, Ross MI, et al. Contemporary diagnostic imaging modalities for the staging and surveillance of melanoma patients: a meta-analysis. J Natl Cancer Inst 2011; 103: $129-42$.

21. Catalano O, Siani A. Cutaneous melanoma: role of ultrasound in the assessment of locoregional spread. Curr Probl Diagn Radiol 2010; 39: 30-6.

22. Schmid-Wendtner MH, Burgdorf W. Ultrasound scanning in dermatology. Arch Dermatol 2005; 141: 217-24.

23. Maj M, Warszawik-Hendzel O, Szymanska E, et al. High frequency ultrasonography: a complementary diagnostic method in evaluation of primary cutaneous melanoma. G Ital Dermatol Venereol 2015; 150: 595-601.

24. Crisan M, Crisan D, Sannino G, et al. Ultrasonographic staging of cutaneous malignant tumors: an ultrasonographic depth index. Arch Dermatol Res 2013; 305: 305-13.

25. Guitera P, Li LX, Crotty K, et al. Melanoma histological Breslow thickness predicted by $75-\mathrm{MHz}$ ultrasonography. Br J Dermatol 2008; 159: 364-9. 